Key messages

- Place of residence may affect health, and mortality from most common diseases tends to be higher in areas characterised by low socioeconomic position

- Research dating back over 100 years suggests that social fragmentation may influence suicide

- In the 1980s and 1990s, parliamentary constituencies with high levels of social fragmentation had high rates of suicide, independent of deprivation

- Constituencies with the greatest increases in social fragmentation between 1981 and 1991 also had the greatest increases in suicide rates over the same period

- Any targeting of suicide prevention may be more effective if aimed at socially fragmented rather than deprived areas

social fragmentation index ${ }^{12}$ might be updated to reflect social trends-for example, the proportion of unmarried people could be replaced by the proportion currently divorced or separated, to reflect increasing cohabitation.

Contributors: Study designed followed discussion between EW, DG, DD, and GDS. EW carried out the analyses and, with DG, wrote a preliminary draft of the paper. All authors contributed to writing the final draft. EW and DG will act as guarantors.
Funding: None.

Competing interest: None declared.

1 Davey Smith G, Hart C, Watt G, Hole D, Hawthorne V. Individual social class, area-based deprivation, cardiovascular disease risk factors, and mortality: the Renfrew and Paisley study. J Epidemiol Community Health 1998;52:399-405.

2 Carstairs V.Deprivation indices: their interpretation and use in relation to health.J Epidemiol Community Health 1995;49(suppl 2):S3-8.

3 Davey Smith G, Dorling D. "I'm all right John": voting patterns and mortality in England and Wales, 1981-92. BMJ 1996;313:1573-7.

Phillimore P, Beattie A, Townsend P. Widening inequality of health in northern England, 1981-91. BMJ 1994;308:1125-8.

5 Davey Smith G, Neaton JD, Wentworth D, Stamler J. Socioeconomic differentials in mortality risk among men screened for the multiple risk factor intervention trial. 1. White men. Am J Pub Health 1996:86:486-96.

6 Gunnell D, Peters T, Kammerling M, Brooks J. The relation between parasuicide, suicide, psychiatric admissions, and socioeconomic deprivation. BMJ 1995;311:226-30

7 Bunting J, Kelly S. Geographic variations in suicide mortality, 1982-1996. Pop Trends 1998;93:7-18.

8 Ashford JR, Lawrence PA. Aspects of the epidemiology of suicide in England and Wales. Int J Epidemiol 1976;5:133-44.

9 Saunderson T, Haynes R, Langford IH. Urban-rural variations in suicides and undetermined deaths in England and Wales. J Pub Health Med 1998;20:261-7.

10 Durkheim E. Suicide: a study in sociology [translation by Spalding J, Simpson G]. London: Routledge and Kegan Paul, 1952.

11 Sainsbury P. Suicide in London. London: Chapman and Hall, 1955.

12 Congdon P. Suicide and parasuicide in London: a small-area study. Urban Studies 1996;33:137-58.

13 Lester D. A regional analysis of suicide and homicide rates in the USA search for broad cultural patterns. Soc Psychiatry Psychiatr Epidemiol 1988;23:202-5.

14 Davey Smith G, Dorling D. Associations between voting patterns and mortality remain. BMJ 1997;315:430-1.

15 Charlton J, Kelly S, Dunnell K, Evans B, Jenkins R, Wallis R. Trends in suicide deaths in England and Wales. Pop Trends 1992;69:10-6.

16 Townsend P, Phillimore P, Beattie A. Health and deprivation: inequality and the north. London: Croom Helm, 1988.

17 Charlton J. Trends and patterns in suicide in England and Wales. Int J Epidemiol 1995;24:S45-52

18 MacIntyre S, MacIver S, Sooman A. Area, class and health: should we be focusing on places or people? J Soc Pol 1993;22:213-34.

(Accepted 6 June 1999)

\title{
Does publicity about cancer screening raise fear of cancer? Randomised trial of the psychological effect of information about cancer screening
}

\author{
Jane Wardle, Tamara Taylor, Stephen Sutton, Wendy Atkin
}

Critics of cancer screening have suggested that the publicity associated with it can provide people with a new health worry. ${ }^{1}$ After a mass media heart disease campaign in Norway a national survey showed that $17 \%$ of those who had seen the campaign materials were worried about heart disease and that these people were most likely to make behaviour changes. ${ }^{2}$ No comparable data on publicity about cancer screening are available. ${ }^{3}$

We evaluated the impact of publicity about a new bowel cancer screening programme, comparing a group who had been sent information about the programme with a control group who had not.

\section{Participants, methods, and results}

Participants aged 55-64 were identified from family health services authority registers and confirmed by their general practitioner to be suitable for screening for bowel cancer. This study group is part of a randomised controlled trial of flexible sigmoidoscopy for the prevention of bowel cancer.

Altogether 2961 adults in Leicester were randomised on a 2:1 ratio, with computer generated random allocations, to receive brief information by post about the flexible sigmoidoscopy screening test $(n=1974)$ or not $(n=987)$. Couples were randomised together to avoid contamination. People in the information group were also asked whether they would be interested in having the test. All participants were sent a letter from their general practitioner requesting their cooperation with the questionnaire study and a questionnaire, which included items on worry about bowel cancer, perceived risk, minor bowel symptoms, and anxiety (shortened version of the state trait anxiety inventory). Demographic and health status items were also included (table). Participants were unaware that they were participating in a study of the effect of information. The study was powered to detect a 0.6
ICRF Health

Behaviour Unit, Department of Epidemiology and Public Health, University College London, London WC1E 6BT Jane Wardle professor Tamara Taylor research psychologist Stephen Sutton senior scientist ICRF Colorectal Cancer Unit, St Mark's Hospital, Harrow, Middlesex Wendy Atkin deputy director

Correspondence to: Professor Wardle j.wardle@ucl.ac.uk

BMJ 1999;319:1037-8 
Demographic characteristics of participants in study, and attitudes to bowel cancer

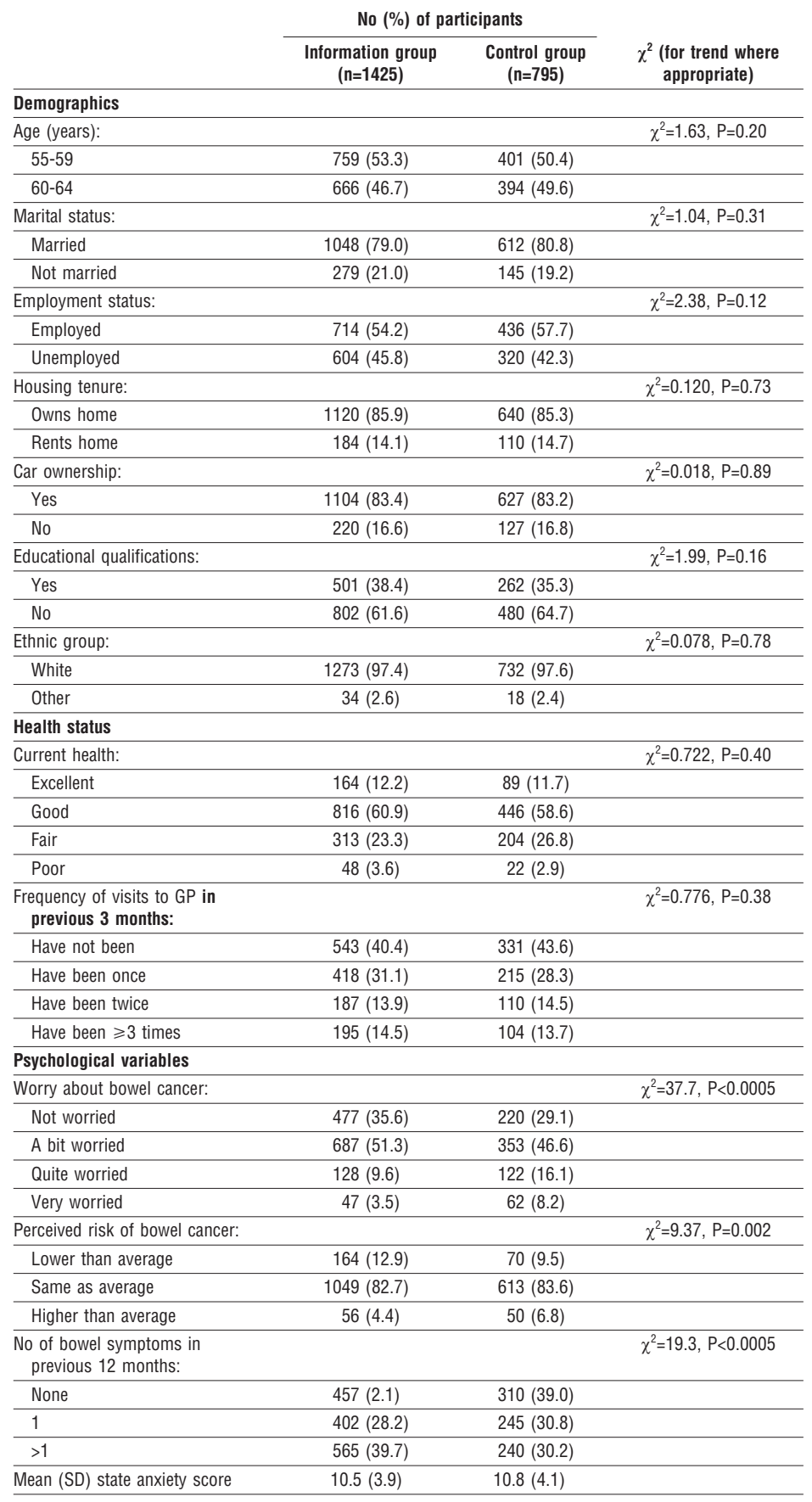

$\mathrm{GP}=$ general practitioner.
$(35.6 \%$ $29.1 \%$ said they were not at all worried; $95 \%$ confidence interval for difference $2.4 \%$ to $11 \%$ ), as was perceived risk $(4.4 \%$ v $6.8 \%$ perceived their risk to be higher than average $(0.3 \%$ to $4.6 \%))$. There was a trend for anxiety to be lower in the intervention group (10.5 $v 10.8 ; t=1.7, \mathrm{P}=0.09 ;-0.66$ to 0.05 ). The information group reported more minor bowel symptoms in the previous three months than the control group $(32.1 \%$ v $39.0 \%$ reported no symptoms; $-11 \%$ to $-2.7 \%)$. The pattern of results was the same when analysed separately for men and women.

\section{Comment}

Publicity about screening did not increase either worry about bowel cancer or subjective risk; indeed, the information seemed to be reassuring, with the informed group reporting less worry and lower subjective risk. The only evidence of any adverse effect was that patients in the information group were more likely to report minor bowel symptoms. This might be attributable to the recognised phenomenon of an increased perception of symptoms while thinking about a particular disease. ${ }^{5}$

An alternative explanation for the apparently reassuring effect of screening is that the information caused denial-respondents were more worried but would not admit it. This seems unlikely, given that the information group reported more symptoms than the control group. The response rate was slightly lower in the information group; this raises the possibility of a small subset of anxious non-responders, whose experiences are not reflected in studies that use postal questionnaires to collect the data.

Overall, these results are encouraging. They give no support to the idea that publicity about cancer screening produces widespread alarm, at least in this age group, and even suggest that screening publicity can have a positive effect.

Contributors: JW participated in developing the protocol, designing the questionnaire, and writing of the paper. TT set up the database, analysed the data, conducted the literature search, and participated in writing the paper. SS participated in developing the protocol and designing the questionnaire. WA contributed to the design and implementation and participated in writing the paper.

Funding: Financial support for the flexible sigmoidoscopy trial was provided by the Imperial Cancer Research Fund and the Medical Research Council.

Competing interests: None declared. anxiety score.

Response rates were high, particularly in the control group $(80.4 \%(794 / 987)$ for the controls $v$ $72.2 \%(1425 / 1974)$ for the information group; $\left.\chi_{1}^{2}=24.5, \quad \mathrm{P}<0.0001\right)$. More women than men responded $(79.1 \%(1169 / 1477)$ v $70.8 \%$ (1051/1484); $\left.\chi^{2}(1)=27.3, \mathrm{P}<0.0001\right)$. There were no significant demographic differences between groups or any differences in health status or visits by the general practitioner (table).

Reported worry about bowel cancer was lower in the information group than the control group
1 Skrabanek P. False premises and false promises of breast cancer screening. Lancet 1985;ii:316-20.

2 Søgaard AJ, Fønnebø V. Self-reported change in health behaviour after a mass media-based health education campaign. Scand J Psychol 1992;33:125-34.

3 Wardle J, Pope R. The psychological costs of screening for cancer. J Psychosom Res 1992;36:609-24.

4 Atkin WS, Hart A, Edwards R, McIntyre P, Aubrey R, Wardle J, et al. Uptake, yield of neoplasia, and adverse effects of flexible sigmoidoscopy screening. Gut 1998;42:560-5.

5 Woods SM, Natterson J, Silverman J. Medical students' disease: hypochondriasis in medical education. J Med Educ 1966;41:785-90.

(Accepted 29 July) 